\title{
Seguridad e inmunogenicidad de la vacuna oral contra el cólera: estudio en 20 voluntarios
}

\author{
Elizabeth Castañeda', Magaly Chinchilla', Martha Velandia², Fernando de la $\mathrm{Hoz}^{2}$
}

\begin{abstract}
Resumen
Se realizó un estudio de seguridad e inmunogenicidad de la vacuna de células enteras de Vibrio cholerae 01 más la subunidad $\mathrm{B}$ recombinante de la toxina ( $\mathrm{CE} / \mathrm{sBr}$ ) en 20 voluntarios sanos, en el Laboratorio de Microbiología del Instituto Nacional de Salud. Se suministraron tres dosis de la vacuna con intervalos de dos semanas entre cada una y se recolectaron muestras de sangre el día de la primera dosis y semanalmente durante ocho semanas. Se anotaron los efectos secundarios durante los cinco días siguientes a la ingestión de cada una de las dosis. En los sueros, se determinaron los niveles de anticuerpos antibacterianos empleando la técnica vibriocida y los de anticuerpos antitoxina de las clases $\lg G$ e $\lg A$ empleando la técnica de ELISA. Se presentaron efectos secundarios leves como náuseas, cefalea y malestar abdominal después de cada una de las dosis en 4, 5 y 2 de los voluntarios. El porcentaje de seroconversión más alto para títulos vibriocida fue del $50 \%$ y la mayor media geométrica fue de $21,38(15,3-29,85)$ ambos eventos en la tercera semana. El porcentaje máximo de seroconversión para los anticuerpos antitoxina fue del $84 \%$ en la octava semana para lgG y del $89 \%$ en la sexta semana para IgA y la media geométrica fue de 245,5 (198-305) en la sexta semana para IgG y de $74,13(60-98)$ en la tercera semana para IgA. Con estos datos podemos concluir que la vacuna $\mathrm{CE} / \mathrm{sBr}$ fue segura e inmunogénica en los voluntarios estudiados.
\end{abstract}

\section{Summary}

The safety and immunogenicity of a three-dose course of oral recombinant B subunit whole-cell cholera vaccine (rBS/WC) was evaluated on 20 healthy volunteers from the Instituto Nacional de Salud. Participants received three doses, separated by two-week intervals. Blood was taken on the day of the first dose administration, and weekly during the following eight weeks. Side effects were registered during the 5 days following the ingestion of each dose. Sera were tested for antibacterial antibodies using vibriocidal techniques and antitoxin antibodies (class $\lg G$ and $\lg A$ ) using the ELISA test. Side effects such as nausea, headache and abdominal discomfort were negligible. The highest seroconversion percentage $(50 \%)$ for antibacterial antibodies when vibriocidal techniques were employed, and the highest geometric mean titer (GMT 21.38) were obtained three weeks after the administration of the first dose. Seroconversion for antitoxin antibodies was obtained in $84 \%(\operatorname{lgG})$ and $89 \%(\operatorname{lgA})$ of the participants at eight and six weeks, respectively. A three-dose course of the oral vaccine ( $\mathrm{rBS} / \mathrm{WC}$ ) was found to be safe and immunogenic in the population studied.

${ }^{1}$ Grupo de Microbiología, INS.

${ }^{2}$ Grupo de Vigilancia Epidemiológica, INS. 
Vibrio cholerae 01 tiene la capacidad de producir una toxina, la cual es una proteína compuesta de dos subunidades: la subunidad A constituye la parte toxigénica y la subunidad $B$ la parte inmunogénica. Esta última subunidad se une al gangliósido de la membrana del enterocito y permite la entrada de la subunidad $A$ al citoplasma, lo cual incrementa la producción de $\mathrm{AMPc}$ lo que conduce a una alteración de la absorción de sodio y cloro e induce la secreción de bicarbonato (1).

En la infección por $V$. cholerae 01 , se induce una respuesta antibacteriana contra el lipopolisacárido y las proteínas de membrana y una respuesta antitóxica contra la subunidad B de la toxina; en esta respuesta desempeña un papel importante la IgA secretora (2). Los anticuerpos antibacterianos son principalmente de la clase lgM; éstos se producen en los primeros días de la exposición, alcanzan sus niveles máximos en la primera semana y desaparecen en un período de seis meses. Los anticuerpos antitoxina son de la clase IgG e IgA y se mantienen por un período de dos años $(1,3,4)$. Además, en la mucosa intestinal se crea una memoria inmunológica, lo cual explica el largo tiempo de protección después de la enfermedad, que evita la reinfección en un $90 \%$ de los casos (5). Este hallazgo ha sido la base para el desarrollo de las vacunas orales como una de las medidas de control del cólera (3).

En la actualidad se han descrito y estudiado varias posibles vacunas orales contra el cólera; una de ellas es la compuesta por células enteras de $V$. cholerae 01 más la subunidad $B$ recombinante de la toxina $(\mathrm{CE} / \mathrm{sBr})(1)$. Esta vacuna posee múltiples estudios de fase II, en los cuales se ha determinado la seguridad y la inmunogenicidad, incluyendo uno realizado en Barranquilla, Colombia (6), y un estudio de fase III, de eficacia protectora, en Bangladesh (7). Otra posible vacuna es la compuesta por células vivas de una mutante de V. cholerae 01, denominada CVD 103 $\mathrm{HgR}$; la cepa tiene una deleción del gen que codifica para la subunidad A de la toxina, lo cual baja su patogenicidad; con esta vacuna se han realizado varios estudios de fase II y actualmente se está realizando un estudio de eficacia en Jakarta, Indonesia (8-10).
El propósito de este trabajo fue determinar, en pequeña escala y en paralelo con el ensayo de campo realizado en Barranquilla, la seguridad e inmunogenicidad de la vacuna $\mathrm{CE} / \mathrm{sBr}$ y describir la cinética de la respuesta humoral producida después de la ingestión de tres dosis de esta vacuna en voluntarios adultos.

\section{Materiales y métodos}

Voluntarios: participaron en el estudio 20 personas sanas, en edades comprendidas entre los 20 y 45 años, las cuales dieron su consentimiento.

Vacuna: se utilizó la vacuna compuesta de células enteras (CE) de tres cepas de Vibrio cholerae 01: Cairo 48 (subtipo Inaba, biotipo clásico), Filip 6973 (Inaba, El Tor), Cairo 50 (Ogawa, clásico), inactivadas por calor o formol más la subunidad $B$ recombinante de la toxina $(\mathrm{sBr})$ obtenida por ingeniería genética. La vacuna utilizada fue suministrada por el Laboratorio de Bacteriología de Suecia y formaba parte del lote empleado para el estudio de seguridad e inmunogenicidad realizado en Barranquilla (6).

Administración de la vacuna: se administraron oralmente tres dosis de la vacuna en las semanas 0,3 y 5 . Cada dosis consistía de $3 \mathrm{~mL}$ diluidos en $150 \mathrm{~mL}$ del antiácido (Samarín), el cual está compuesto por bicarbonato de sodio, ácido tartárico, ácido cítrico, carbonato de sodio y tartrato sódico de potasio.

Vigilancia de efectos secundarios: se repartió a cada voluntario un formulario que indagaba por la presencia de fiebre, cefalea, malestar, náuseas, vómito, dolor abdominal y diarrea, esta última definida como la presencia de tres o más deposiciones blandas o líquidas en $24 \mathrm{~h}$ durante los 7 primeros días después de cada una de las dosis.

Serología: se recolectaron $5 \mathrm{~mL}$ de sangre, semanalmente en las semanas 0 a 8 , a partir de la primera dosis de la vacuna. En el suero se determinó la presencia de anticuerpos antibacterianos por medio de la microtécnica vibriocida realizada con la cepa de $V$. cholerae 01 subtipo Inaba (T19479) y complemento de cobayo (Sigma). El título del suero fue considerado como la mayor dilución de éste que inhibía 
completamente el crecimiento bacteriano; se consideró como seroconversión un incremento igual o mayor a dos diluciones entre la muestra de la semana 0 y las muestras de cada una de las siguientes semanas (11).

Los títulos de anticuerpos antitoxina lgG e lgA se determinaron por medio de una técnica de ELISA, empleando como antígeno la subunidad $B$ de la toxina unida al gangliósido GM1 (Sigma) en placas de baja afinidad (Immulon 1, Dynatech). Se consideró como seroconversión un incremento del título de anticuerpos igual o mayor 1,5 veces con respecto al título de la semana 0 (12).

Métodos estadísticos: inicialmente se obtuvo la media geométrica de los títulos de anticuerpos vibriocidas, antitoxina IgG e IgA por medio de la transformación logarítmica de los títulos, metodología propuesta por Paul y White (13). Los promedios se compararon usando la prueba t de Student para muestras apareadas; el nivel de confianza usado para las comparaciones fue del $95 \%$.

\section{Resultados}

Efectos secundarios: los 20 voluntarios llenaron el formulario de vigilancia de efectos secundarios. Con la primera y segunda dosis (semana 0 y 3 ) un total de 6 voluntarios manifestaron algún efecto colateral, 2 dijeron haber presentado cefalea, 1 dolor abdominal, 1 malestar abdominal y 2 náuseas. Los efectos secundarios disminuyeron notablemente con la tercera toma. Ninguno de los voluntarios requirió tratamiento médico o incapacidad.

Respuesta inmune: se tomaron 175 muestras de sangre distribuidas así: 20 muestras las semanas 0 a $4 ; 18$ en la quinta semana y 19 muestras en las semanas 6 a 8 . El $50 \%$ de los vacunados seroconvirtieron en la prueba vibriocida en la semana 3 . La mayor media geométrica de los títulos vibriocidas se determinó en la tercera y quinta semana y fue de 21,38 (rango: 15,3-29,85), posteriormente, se presentó una leve disminución a partir de la sexta semana hasta la octava semana (figura 1).
En los anticuerpos antitoxina lgG se encontraron diferencias significativas en los porcentajes de seroconversión entre las semanas 5 y 7 comparados con la muestra prevacunal; el mayor porcentaje de seroconversión $(84 \%)$ ocurrió en la octava semana. Los anticuerpos antitoxina IgA se determinaron en la sexta semana en el $89 \%$ de los voluntarios (tabla 1).

La mayor media geométrica de los títulos de lgG antitoxina fue de 245,5 (rango 198-305), obtenida en la séptima semana y se mantuvo hasta la última medición (figura 2). La de IgA fue de 74,13 (rango 60-98) en la tercera semana y a partir de esta disminuyó progresivamente (figura 3 ).

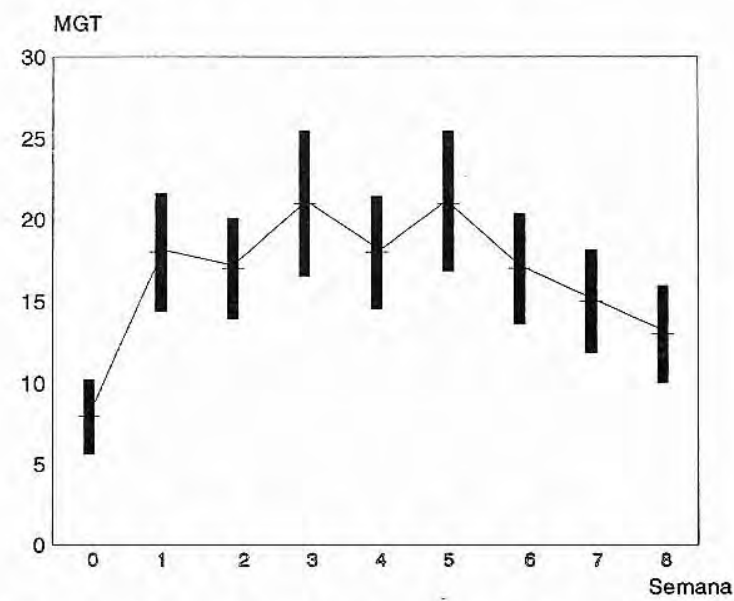

Figura 1. Media geométrica de los títulos vibriocidas por semana.

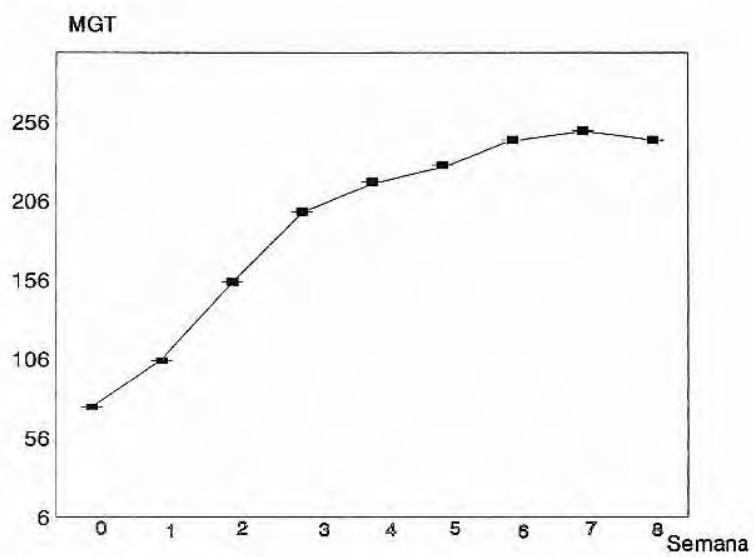

Figura 2. Media geométrica de los títulos de anticuerpos lgG antitoxinas por semana. 
Tabla 1. Porcentajes de seroconversión por semana para las antitoxinas $\lg G$ e $\lg A$.

\begin{tabular}{crrrrrrrr}
\hline & \multicolumn{10}{c}{ Semanas } \\
\cline { 2 - 9 } & 1 & 2 & 3 & 4 & 5 & 6 & 7 & 8 \\
\hline AT IgG & 32 & 50 & 60 & 70 & 80 & 83 & 79 & 84 \\
AT IgA & 47 & 67 & 80 & 80 & 85 & 89 & 84 & 74 \\
\hline $\mathrm{n}$ & 20 & 20 & 20 & 20 & 18 & 19 & 19 & 19 \\
\hline
\end{tabular}

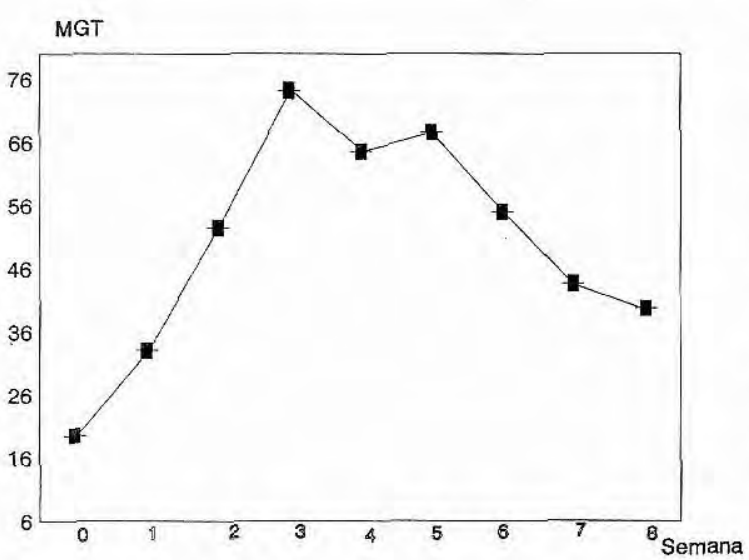

Figura 3. Media geométrica de los títulos de anticuerpos IgA antitoxina por semana.

\section{Discusión}

La vacuna anticólera empleada en este estudio, compuesta de células muertas más la subunidad $B$, fue empleada en Bangladesh en estudios de campo de fase III y mostró una eficacia protectora del $65 \%$ a un año y $52 \%$ a tres años de seguimiento $(7,14)$. Dado que las características inmunológicas difieren entre poblaciones endémicas y epidémicas, era necesario llevar a cabo, en América Latina, estudios previos a la aplicación masiva de la vacuna, que reflejaran su seguridade inmunogenicidad (8).

Sólo seis de los 20 voluntarios encuestados manifestaron algún síntoma, todos clasificados como leves, confirmándose con ello la seguridad de la vacuna en este grupo. En el estudio realizado en Bangladesh, la presencia de síntomas colaterales después de la vacunación fue atribuida al antiácido en el cual se disuelve la preparación para proteger la subunidad B de la toxina de la acidez gástrica (15). Los hallazgos en el ensayo de campo en Barranquilla y los de este estudio apoyan la hipótesis del antiácido como el principal causante de los efectos secundarios (6).

El porcentaje de seroconversión del 50\% para anticuerpos vibriocidas obtenido en este estudio fue similar a los resultados del $49 \%, 45 \%$ y $52 \%$ obtenidos en Bangladesh, Tailandia y Suecia, respectivamente, pero menor que el $89 \%$ informado en voluntarios norteamericanos, empleando en todos estos casos la misma preparación (15-18). El estudio de fase II realizado en Barranquilla utilizando el mismo lote de la vacuna oral $\mathrm{CE} / \mathrm{sBr}$ demostró un porcentaje de seroconversión para anticuerpos vibriocidas del $44 \%$, lo cual correlaciona con el obtenido en este estudio (6).

Los resultados de anticuerpos vibriocidas permiten sugerir que, de realizarse un ensayo de eficacia de esta vacuna en nuestro país, los resultados de este serían similares al $60 \%$ obtenido en Bangladesh, dado que la seroconversión para vibriocida es el mejor predictor de protección clínica (2).

Es de anotar que el porcentaje de seroconversión hallado en otro estudio, también en voluntarios colombianos, pero con la posible vacuna oral CVD $103 \mathrm{HgR}$, fue mayor del $80 \%$ (19). Esto hace pensar que la eficacia de esta segunda vacuna podría ser mejor que la de $\mathrm{CE} / \mathrm{sBr}$. La respuesta definitiva sólo la daría un ensayo de campo midiendo la eficacia de ambas vacunas; pero, fisiológicamente es de esperarse que una vacuna con células vivas, las cuales conservan su capacidad para reproducirse en la luz intestinal, produzca una respuesta vibriocida mayor $y$, por tanto, un mejor efecto protector.

En estudios previos de la vacuna en Bangladesh y voluntarios suecos, se informaron porcentajes de seroconversión de los títulos de anticuerpos de antitoxina $\lg G$ e $\lg A$ similares a los encontrados en este estudio $(16,18)$. En otros ensayos en poblaciones tailandesas y norteamericanas, se informaron porcentajes de seroconversión para IgG más altos que el obtenido en este estudio (17). 
También, en Barranquilla, se encontraron cifras de seroconversión para IgG e IgA ( $88 \%$ y $80 \%)$ que eran semejantes a los hallados en la presente investigación. Esta comparación es cierta parcialmente, dado que en Barranquilla sólo se tomaron muestras hasta la cuarta semana y en este estudio se tomaron semanalmente hasta la octava semana. Otra diferencia importante en el presente estudio fue que se aplicaron tres dosis contra sólo dos administradas en Barranquilla; esto se tradujo en un aumento importante de la seroconversión para $\operatorname{lgG}$ e $\lg A$ a partir de la tercera dosis, manteniéndose estos niveles elevados hasta el fín del seguimiento. El mismo comportamiento no fue observado con los anticuerpos vibriocidas, los cuales disminuyeron a partir de la cuarta semana, lo cual sugiere que la dosis extra no presenta beneficios (6).

En resumen, el estudio demuestra que la seguridad e inmunogenicidad de la vacuna en voluntarios colombianos es similar a las observadas en la mayoría de los estudios previos $(7,14$ 18). La decisión de una vacunación masiva; sin embargo, debe ser tomada con base en la eficacia lograda en poblaciones similares y evaluando el costo-beneficio de esta intervención.

\section{Agradecimientos}

A los voluntarios por participar en este estudio.

\section{Referencias}

1. Holmgren J, Clemens J, Sack DA, et al. New cholera vaccines. Vaccine 1989;7:94-6.

2. Svennerholm AM, Jonson G, Holmgren J. Immunity to Vibrio cholerae infection. Chapter 16. In: Wachsmuth IK, Blake PA, Olsvik 0, editors. Vibrio cholerae and cholera. Washington, D.C.: American Society of Microbiology, 1994:258-60.

3. World Health Organization. Development of vaccines against cholera and diarrhoea due to enterotoxigenic Escherichia coli: memorandum from a WHO meeting. Bull WHO 1990;68:303-12.

4. Levine MM, Herrington D, Losonsky G, et al. Safety, immunogenicity and efficacy of recombinant live oral cholera vaccines, CVD 103 and CVD 103-HgR. Lancet 1988;II:467-70.

5. Svennerholm AM, Jertborn M, Gothefors L, et al. Mucosal antitoxic and antibacterial immunity after cholera disease and after immunization with a combined $B$ subunit-whole cell vaccine. J Infect Dis 1984;149:884-93.

6. Concha A, Giraldo A, Castañeda E, et al. Safety and immunogenicity of oral killed whole cell-recombinant B subunit cholera vaccine in Colombia. WHO Bulletin, aceptado para publicación, 1994.

7. Clemens JD, Harris JR, Sack DA, et al. Field trial of oral cholera vaccines in Bangladesh: results of one year of follow-up. J Infect Dis 1988;158:60-9.

8. Organización Panamericana de la Salud. Evaluación de las vacunas contra el cólera. Boletín epidemiológico 1991;12:11-4.

9. Kaper JB. Vibrio cholerae vaccines. Rev Infect Dis 1989;11:s568-s573.

10. Sack RB, Albert MJ. Cholera vaccine workshop. J Infect Dis 1994;170:256.

11. Benenson AS, Saad M, Mosely WH. Serological studies in cholera. 2. The vibriocidal antibody response of cholera patients determined by microtechnique. Bull $\mathrm{WHO}$ 1968;38:277-285.

12. Holmgren J, Svennerholm AM. Enzyme-linked immunoabsorbent assays for cholera serology. Infect Immun 1973;7:759-63.

13. Paul E, White F. Seroepidemiology. New York: Oxford Press, 1954.

14. Clemens JD, Sack DA, Harris JR, et al. Field trial of oral cholera vaccines in Bangladesh: results from three-year follow-up. Lancet 1990;।:270-4.

15. Sánchez JL, Trofa AF, Taylor DN, et al. Safety and immunogenicity of the oral, whole cell/recombinant B subunit cholera vaccine in Northamericans volunteers. $\mathrm{J}$ Infect Dis 1993;167:1446-9.

16. Sack DA, Clemens JD, Huda S, et al. Antibody responses after immunization with killed oral cholera vaccines during the 1985 vaccine field trial in Bangladesh. J Infect Dis 1991;164:407-11.

17. Pitisuttithum $P$, Migasena $S$, Suntharasamai $P$, et al. Immune responses following killed whole vibrio-B subunit oral cholera vaccine in human volunteers. South Asian J Trop Med Pub Health 1989;20:201-5.

18. Jertborn M, Svennerholm AM, Holmgren J. Safety and immunogenicity of an oral recombinant cholera B-subunit whole cell vaccine in Swedish volunteers. Vaccine 1992;10:130-2.

19. Castañeda E, Chinchilla M, Velandia M, de la Hoz F. Seguridad e inmunogenicidad de la vacuna oral anticólera CVD-103 HgR: estudio en 29 voluntarios. Biomédica 1995; 15:54-58. 